\title{
Pérdida de Oportunidad
}

\section{Loss of opportunity}

\section{SAMUEL YONG \\ Profesor de la Facultad de Derecho de la Universidad Santo Tomás. Correo electrónico:yonghernandez@yahoo.es \\ Professor at the Law Faculty of the Santo Tomas University. E-mail: yonghernandez@yahoo.es \\ CAMILO A. RODRÍGUEZ YONG Profesor de la Facultad de Derecho de la Universidad del Rosario Professor at the Law Faculty of the Santo Tomas University.}

Fecha de recepción: 9 de junio de 2011 Fecha de evaluación: 10 de junio de 2011 Fecha de aprobación: 15 de junio de 2011

Resumen. El presente trabajo tiene por objeto describir las diferentes formas como se viene aplicando y tratando la figura de la pérdida de oportunidad con el fin de acercarnos al estudio de la misma.

Palabras clave. Probabilidad, pérdida, ventaja, chance, oportunidad, incertidumbre, nexo causal 
Abstract. The current work has as an objective to describe the different ways how the doctrine of loss of chance is being applied and treated, with the purpose of providing an approach to its study.

Key words. Probability, lost, chance, oportunity, uncertainty, causal nexus.

Tipo de Artículo: Reflexión Académica

SUMARIO: 1. Introducción 2. Origen 3. Teoría negativa y positiva sobre la pérdida de una oportunidad 4. Conclusiones.

\section{Introducción}

Existen un sinnúmero de situaciones en las que un sujeto tiene la probabilidad de obtener una ventaja o evitar una perdida, pero por hechos provenientes de un tercero esa oportunidad se malogra. ¿Será ese tercero responsable, cuando con su conducta activa u omisiva le frustra la esperanza a quien posiblemente se beneficiaría de un resultado, que aunque aleatorio,-- pierde definitivamente la oportunidad de alcanzarlo? Así, ¿ debería indemnizarse a una persona por 
habérsele frustrado la oportunidad de contraer matrimonio debido a lesiones causadas con arma de fuego que le generaron problemas de orden sexual o desfiguración facial?; ¿o a los padres de hijos fallecidos- tempranamente, que no pudieron obtener la ayuda económica de estos una vez alcanzaran la edad de trabajar?; ¿o a un enfermo que pierde una posibilidad de curación por una mala praxis médica?; ¿o a una de las partes de un proceso judicial que en segunda instancia no tuvo la oportunidad de revocar una decisión adversa porque su apoderado no interpuso el respectivo recurso?; ¿o a una candidata en un concurso de belleza que, opcionada para ganarlo, no fue informada que había quedado entre las semifinalistas y por tal razón no siguió participando?; ¿0 al oferente de una licitación que no pudo seguir participando por un infundado rechazo de su propuesta?; ¿o al jugador de un equipo profesional de fútbol que encontrándose en negociaciones serias con un club europeo, pierde un pie como consecuencia de un accidente -automovilístico?, etc.

Tanto la jurisprudencia como la doctrina han tratado de darle respuesta a estas realidades caracterizadas por la dificultad para apreciar la certeza de un perjuicio (Mazeaud-Tunc, p._307). Para ello, han acudido a la figura de la pérdida de oportunidad, cimentada generalmente en la teoría ontológica y desarrollada en sus diferentes matices (daño patrimonial o personal) y vertientes (teoría ontológica moderada y teoría ontológica radical). 
La oportunidad perdida ha sido objeto de un encendido debate. Algunos la niegan como sucede con los seguidores - de la teoría del todo o nada; otros, sí bien la aceptan, tienen diferencias sobre la forma como se debe dar respuesta al problema. Así, dejando a un lado la teoría ontológica y con fundamento en la doctrina de la causalidad probabilística, ven la pérdida de una oportunidad no como la afectación de un bien en sí diferente del perjuicio final, sino como un sistema alternativo de imputación, válido para casos de incertidumbre causal.

Lo anterior, aunado a otras diferencias que se reflejan entre los mismos partidarios de la aplicación de la pérdida de oportunidad, nos ha llevado a realizar este escrito con el fin de acercarnos al estudio de esta figura; definida por algunos como la "oportunidad verosímil de lograr una ventaja o de impedir una pérdida" (Zavala, p. 402).

\section{Origen}

De acuerdo con Medina Alcoz, la doctrina francesa hace suya la teoría de la pérdida de oportunidad, sin embargo, otros ordenamientos jurídicos se han ocupado de esta figura. Se pueden encontrar antecedentes a principios del siglo XIX en Francia, pero también en los tribunales británicos y norteamericanos (Estados Unidos y Canadá) (p. 115 B). En Francia, los primeros casos se relacionaron con negligencias de profesionales forenses mientras que en el 
Reino Unido, el primer caso tuvo que ver con un concurso de belleza (Ibíd., p, 116).

Posteriormente, la doctrina de la pérdida de oportunidad fue cobrando más importancia y extendiéndose a otros ordenamientos, así como a organizaciones europeas e internacionales. Al respecto el profesor Medina Alcoz comenta: "la teoría ha circulado horizontalmente, penetrando otros muchos ordenamientos nacionales (Australia, Bélgica, Holanda, Italia, Argentina, entre otros); y verticalmente, alcanzando a organizaciones europeas e internacionales de diverso signo (Consejo y Tribunal de Justicia de las Comunidades Europeas; Tribunal Europeo y Corte Interamericana de Derechos Humanos; Instituto Internacional para la Unificación del Derecho Privado -UNIDROIT-; Academia de Jusprivatistas Europeos; Grupo Europeo que, después de identificarla a través del método comparado, la han recogido o aplicado en algunos de sus instrumentos (Art. 2.7 Directiva 92/13/CE y jurisprudencia comunitaria; sentencias en materia de derechos humanos; Art. 7.4.3 Principios UNIDROIT; Art. 163 Parte General del Código Europeo de Contratos; Art. 3:106 Principios del Derecho Europeo de la Responsabilidad Civil)" (Ibíd., ps.115 y 116).

Con todo, debe señalarse que hay países como Alemania, Austria, Dinamarca, Finlandia, Noruega, Suecia y Suiza donde la jurisprudencia no aplica la teoría de la perdida de oportunidad, por considerar que esta no es susceptible de reparación (Medina, p. 138 A); incluso en los Estados Unidos, país pionero en el tema, sólo 
algunos estados aceptan la teoría y exclusivamente para casos relacionados con responsabilidad médica ${ }^{1}$. En esta área particular, la doctrina y la jurisprudencia norteamericana han identificado varios argumentos a favor de su adopción. Uno de estos es que si las cortes la rechazaran, un médico escaparía de responsabilidad aún en el caso de la más grave negligencia, si el paciente tuviese menos del $50 \%$ de posibilidades de sobrevivir. Otro argumento es que sin importar que tan pequeño es el chance del paciente de recuperarse o sobrevivir, la pérdida de este tiene un valor que debe indemnizarse. (Wallace, ps 223 y 224). Y un tercer argumento es que la falta de reconocimiento al valor de una oportunidad pondría al paciente a soportar las consecuencias derivadas de la negligencia del médico, quien es la parte que está en capacidad de evitarla².

Desde una perspectiva general, la admisión de una doctrina sobre la pérdida de un chance obedeció a razones de equidad, ya que la injusticia del nexo de causalidad del todo o nada (Karcher, p. 90) dejaba sin indemnización muchos casos que eran dignos de ser indemnizados. En este sentido, la teoría de la pérdida de oportunidad procuraba "una solución equilibrada acorde con los dictados de la justicia a la que repugna que se exonere al agente (posiblemente) dañoso por las dificultades probatorias, pero también que se le obligue a reparar la

\footnotetext{
1 "The Oklahoma Supreme Court has determined that only medical malpractice claims qualify for the reduced causation standard entailed in a loss of chance action: "[A]n action for loss of chance of survival may not be expanded to apply in an ordinary negligence action brought against one other than a medical practitioner or hospital". Frey v. AT\&T Mobility, LLC, United States Court of Appeals for the Tenth Circuit, 379 Fed. Appx. 727; Véase igualmente Matsuyama vs. Birnbaum, Supreme Judicial Court of Massachusetts, 452 Mass. 1. Las razones que se esgrimen para limitar su aplicación en el derecho norteamericano obedecen, de una parte, a que es más fácil en casos de mala práctica médica, que en otros casos de responsabilidad, acceder a evidencia experta para establecer la pérdida de oportunidad; y de otro, se justifica como consecuencia del carácter especial de la relación médico-paciente. Hardy, Jr., v. Southwestern Bell Telephone Company, Supreme Court of Oklahoma, 910 P.2d 1024.
} 
totalidad de un daño que pudo no haber causado" (Medina, p 114 B; Tamayo, T. I, p. 291).

En consonancia con esto, el juez, utilizando las facultades limitadas que se le han otorgado, debe aplicar los principios de justicia para establecer si una persona está obligada a responder por un daño que se le ha imputado (Merryman, ps. 98 y 105), mitigando, ante la dificultad probatoria, la dureza del principio de certidumbre respecto del nexo de causalidad para condenar o exonerar.

Como lo señala el profesor Villey al reflexionar sobre la justicia como finalidad del derecho, la misión del juez ha de ser la de llevar a cabo una "partición de los bienes", que sea justa y proporcional. Así, cuando ha ocurrido un incumplimiento contractual o un delito, que han roto el equilibrio patrimonial entre los involucrados, el papel de la justicia es restablecerlo, asignando a cada quien lo que merece (ps. 80 y 92$)$.

\section{Teorías negativa y positivas sobre la pérdida de una oportunidad}

Existen tesis a favor y en contra de las indemnizaciones por pérdida de oportunidad. Mientras una teoría se opone a indemnizaciones de este tipo (teoría del todo o nada relajada o inflexible) las demás teorías las defienden con diferentes matices y vertientes. Entre estas teorías encontramos la teoría 
ontológica del perjuicio patrimonial y del perjuicio personal, según si la privación de la oportunidad recae sobre un bien patrimonial o extrapatrimonial. A su vez, la teoría ontológica del perjuicio patrimonial puede ser moderada o radical, dependiendo del umbral utilizado para categorizar la oportunidad perdida como bien que sale del patrimonio.

Otra teoría que defiende la indemnización de oportunidades perdidas es la teoría de la causalidad probabilística que, al igual que la teoría ontológica del perjuicio patrimonial, tiene dos vertientes, una moderada y otra radical. La moderada sólo admite una indemnización cuando la ventaja estropeada tiene un valor considerable al paso que la radical aboga por indemnizar todas las oportunidades perdidas, sin importar que sean insignificantes.

Por último, hay una teoría que admite la posibilidad de indemnizar oportunidades perdidas pero siempre y cuando no sean casos de responsabilidad médica, pues considera que en estos eventos lo que se presenta es una concurrencia de causas.

\subsection{Teoría negativa sobre la pérdida de oportunidad (teoría del todo o nada)}

La teoría del "todo o nada" rechaza la posibilidad de indemnizar la pérdida de un chance por considerar que va en contravía del fundamento mismo de la 
responsabilidad civil, al permitir el reconocimiento de indemnizaciones en supuestos a los que les falta un requisito, cual es que el hecho dañoso, sin ningún trazo de duda, haya provocado el perjuicio (Medina, p. 325A).

Esgrime asimismo que las teorías positivas sobre pérdida de oportunidad no son congruentes con los eventos que quieren reglar. Ignoran la realidad y por esto llegan a soluciones erróneas, como otorgar una indemnización sin que haya nexo causal que la justifique, o, en casos donde este nexo causal existe, consentir que una indemnización sea parcial cuando debería ser total (lbíd., ps. 325 y 326; Wallace, p. 224).

Por otro lado, estas soluciones son incompatibles con los postulados del análisis económico del derecho, habida cuenta que si se constriñe a un sujeto a indemnizar total o parcialmente un daño que no ha causado, se le impulsa innecesariamente a extremar las medidas de precaución; contradicción que también se ve en el caso de una persona que sí ha causado un daño, pero que sólo debe repararlo en parte, pues se premia parcialmente su negligencia y no se le incentiva a tener mayor cuidado, siendo más lógico lo contrario (Ibíd., ps 326 y 327).

Cabe anotar, que la aplicación de la teoría del todo o nada no es uniforme todo depende del sistema donde se emplee. Según Medina Alcoz, la teoría del todo o nada puede ser con relajación probatoria o con rigurosidad probatoria. En la 
primera, aplicada en el derecho anglosajón, el estándar de certidumbre se encuentra en el 50\%; En la segunda, utilizada en los sistemas de la Europa continental, el umbral de -certeza es del $80 \%$ (ps 327 a 329 A).

A su vez, ambas figuras, pueden calificarse de -exacerbadas, según rebajen o aumenten el umbral del estándar de persuasión antes mencionado; de este modo, si bien se puede reducir aún más el umbral, como sucede en la figura del todo $o$ nada con relajación probatoria exacerbada, se sigue quitando toda relevancia a la teoría de la pérdida de oportunidad, al permitir dulcificar el estándar ordinario de persuasión por debajo del 50\% (common law) ${ }^{31} \mathrm{o}$ del $80 \%$ (derecho europeo continental) para proclamar la existencia del nexo causal entre el hecho dañoso y el daño final, con miras a otorgar una indemnización total en situaciones caracterizadas por la dificultad probatoria, en especial, en los casos de responsabilidad médico- sanitaria. (Ibíd., ps. 330 a 332).

Así mismo, y con más vehemencia, la figura del todo o nada con rigurosidad probatoria exacerbada, aplicada por la jurisprudencia de algunos sistemas de la Europa continental, niega la doctrina de la pérdida de un chance exigiendo una rigurosa acreditación del nexo de causalidad muy superior al estándar ordinario del civil law, esto es, en porcentajes cercanos al 100\%. La jurisprudencia belga ha asumido esta posición (lbíd., ps. 336 y 337).

\footnotetext{
${ }^{3}$ Para nosotros, en el caso norteamericano, creemos que la teoría con relajación probatoria exarcebada, no podría incluirse como una modalidad de la teoría del todo o nada, pues precisamente una de las variantes de la teoría de la pérdida de una oportunidad es la "relaxed causatión approach" la cual permite un umbral menor al 50\% (Véase Borkowski v. Sacheti, 43 Conn. App. 294).
} 


\subsection{Teorías positivas sobre la pérdida de una chance}

Estas teorías tienen en común que admiten la pérdida de una oportunidad, pero se diferencian en algunos aspectos.

\subsubsection{Teoría ontológica o autonomista}

El cambio de paradigma que hubo en el continente, respecto a reconocer que además de los derechos subjetivos, existían otros intereses legítimos, plausibles de amparo ${ }^{4}$, conllevó a que la pérdida de una oportunidad se admitiera más fácilmente y fuera objeto de resarcimiento siempre y cuando se probara; reparación no reconocida en otros tiempos, al ser considerada como un simple daño hipotético o eventual (Calvo, p. 147).

Para algunos partidarios de la aplicación de esta figura, no existe motivo alguno para negar el carácter de daño indemnizable a la pérdida de una chance con el argumento de no poder acreditarse de manera irrefutable la relación causal entre el hecho ilícito y el daño, puesto que el análisis de la certidumbre del daño no puede ser referido al elemento causalidad. La pérdida de oportunidad, posee un valor en sí misma, reduciéndose el problema a establecer cual es su valor (Peirano, p. 369); por tanto, no puede negarse a priori la indemnización de una

\footnotetext{
${ }^{4}$ Definir el daño por el interés que le asiste a la víctima, cobra importancia habida cuenta que el amparo jurídico se puede extender a otras situaciones jurídicas subjetivas, pues basta que aquel "se halle ligado a un bien digno de protección jurídica, aun cuando su titular no posea un medio de protección específico, o revista el carácter de simple o de hecho, es decir, cuando resulte afectada una situación fáctica de provecho -que no cuente a su vez con medios de protección específicos al efecto- de quien detenta una expectativa lícita de continuar disfrutando de dicho estado, siempre y cuando el susodicho interés vulnerado esté investido de licitud y seriedad" (Prévot y Chaia p. 15)
} 
posibilidad pérdida, que pueda ser valorada en sí misma, pues aquella incorpora un daño, con carácter de cierto, no obstante su dificultad para determinar con exactitud su cuantía (Bonasi, ps. 45 y 46; Tamayo, Tomo II, p. 357). La oportunidad perdida comporta el perjuicio de un bien distinto al incorporado en el beneficio esperado, por ende no puede identificarse con el perjuicio final (Medina, p. 349 A).

En este sentido, también algunas cortes norteamericanas han considerado la doctrina de la pérdida de oportunidad, no como una teoría relacionada con la causa, sino como una teoría relacionada con el daño; para ellas, la pérdida de una oportunidad debe reconocerse y valorarse como un daño susceptible de ser compensado. El reconocer la doctrina de la pérdida de oportunidad como una teoría relacionada con el daño no es inconsistente ni elimina el elemento de la causa. El demandante todavía debe demostrar que la negligencia del médico le causó una disminución de la probabilidad de obtener un resultado más favorable 5 .

Es por ello, que para algunos doctrinantes la pérdida de oportunidad sea un daño emergente (Yzquierdo, p. 153; De Cupis, p.319), por cuanto lo indemnizable es la actual posibilidad frustrada de obtener el beneficio (Prevót y Chaia, p. 53) o evitar una pérdida; bienes que se encuentran presentes en el patrimonio de la víctima,

\footnotetext{
${ }^{5}$ Así, en este sentido se ha pronunciado la Suprema Corte de Masachussets: "Recognizing loss of chance as a theory of injury is consistent with our law of causation, which requires that plaintiffs establish causation by a preponderance of the evidence...In order to prove loss of chance, a plaintiff must prove by a preponderance of the evidence that the physician's negligence caused the plaintiff's likelihood of achieving a more favorable outcome to be diminished. That is, the plaintiff must prove by a preponderance of the evidence that the physician's negligence caused the plaintiff's injury, where the injury consists of the diminished likelihood of achieving a more favorable medical outcome. ...The loss of chance doctrine, so delineated, makes no amendment or exception to the burdens of proof applicable in all negligence claims" (Matsuyama vs. Birnbaum Supreme Judicial Court of Massachusetts, 890 N.E.2d 819).
} 
antes de la ocurrencia del hecho dañoso, "bajo la forma de la posibilidad de conseguir un resultado favorable" (Ibíd.); o que otros, la identifiquen con el lucro cesante, el cual será resarcible en la medida en "que el dañado demuestre, aunque sea vía presuntiva, la existencia de un nexo causal entre el hecho y la razonable probabilidad de la verificación futura del daño" (jurisprudencia citada por Prevót y Chaia p. 51).

A partir del reconocimiento de esta figura, puede suceder que un tercero no alcance a ser responsabilizado, cuando de su actuación no se pueda inferir con certidumbre que privó a una persona de la ventaja esperada, pues no habría un nexo de causalidad suficiente entre el hecho y el daño para imputarle su autoría y por consiguiente obligarlo a reparar el perjuicio. Sin embargo, puede acontecer que en esa misma relación la actuación del tercero haya frustrado, no la ventaja o beneficio, sino la posibilidad de obtenerla por parte de quien la esperaba, en este caso, en aplicación de la pérdida de una chance, podría obligarse a ese tercero a reparar parcialmente a la víctima.

De allí, la distinción realizada por la doctrina, entre la pérdida de la ventaja esperada y la privación de las posibilidades de obtenerla (Chabas, p. 65), para inferir que en este último caso nos encontramos en presencia de la pérdida de una oportunidad. 
Para el profesor Chabas, las aleas son en el fondo la cualidad que identifica la figura de la chance, tornándolas en una forma especial de perjuicio. Cuando se hace referencia a la oportunidad, la víctima se encuentra sometida a ella, esperando su ocurrencia; en consecuencia el elemento aleatorio, no es la perdida del proceso judicial sino su resultado favorable, no es mantener una enfermedad sino sanarla, no es la vida sino sobrevivir (p. 69,), no es perder el concurso de belleza o la licitación, sino ganarlo.

En la chance el paso para la pérdida de la ventaja deseada generalmente ya se ha iniciado; por ello, cuando se hace referencia a la perdida de una oportunidad de sobrevivir o de ganar el litigio o la licitación, se está apuntando a aquellas personas que en su caso, no tienen sino oportunidades de no morir, de no perder el pleito o la adjudicación (Ibíd. p. 71).

En este sentido, no podría asimilarse las aleas con el riesgo; de ahí, que cuando una oportunidad se pierde no siempre de lugar a inferir la existencia de una chance. Todos podemos correr el infortunio de estando saludables morir por razones de un tercero o de la naturaleza, y si es por el hecho del tercero, que se nos quita la oportunidad de vivir, no por ello podríamos deducir que estamos en presencia de un caso de pérdida de oportunidad; aquel tercero con seguridad será responsable, pero no en aplicación de esta figura específica, porque en el ejemplo, como lo afirma el profesor Chabas, no tenemos sino oportunidades de morir. El camino que podía conducir a la pérdida de la ventaja esperada no se 
había iniciado, pues no se estaba enfermo o en otra situación que llevara a una probabilidad de pérdida de la vida, y por tanto no se encuentra en juego ningún alea (oportunidad de no morir), es decir, no se ha truncado ninguna probabilidad de sobrevivir, sino la vida en sí. (Ibíd.).

De este modo, la pérdida de una oportunidad se define por la incertidumbre inherente al perjuicio; el beneficio esperado no está garantizado, pues no se puede establecer si llegará, habida cuenta que en su travesía se puede cruzar, para bien o para mal, la mano del hombre o de la naturaleza (lbíd., p. 80).

Y si existe incertidumbre, frente al hecho de no poderse establecer cual es la causa de que el beneficio esperado no se haya obtenido ¿por qué se tiene que indemnizar?

La doctrina, dando respuesta a esta situación, ha señalado que desde el punto de vista de la pérdida de una chance, se presenta una situación en donde coexisten dos elementos aparentemente contradictorios: un elemento de certidumbre respecto del evento que hace malograr la pérdida de la oportunidad de obtener la ventaja esperada, es decir, que si no se hubiese producido el hecho dañoso, la victima habría conservado la expectativa de obtener una ganancia o evitar una pérdida; y otro, de incertidumbre o de duda frente a la situación esperada, pues no se puede determinar si el beneficio, de todas formas, se hubiese obtenido de 
no haberse producido el evento dañoso del tercero ((Trigo, p. 29, Yzquierdo, p. 153, Zannoni, p. 50).

Con todo, a pesar de la situación de incertidumbre, hay algo actual, cierto e indiscutible, como es el hecho de que definitivamente por la actuación del tercero se pierde la oportunidad de lograr un beneficio; en consecuencia, hay lugar a reparación, como quiera que existe un daño cierto, cual es el haber estropeado de manera irremediable la probabilidad que se tenía de alcanzar el beneficio o el de evitar un perjuicio (Trigo, ps. 29 y 30); lo perdido, lo frustrado, en verdad, es la oportunidad perdida y no la ganancia esperada, como tal. (Zannoni, p. 52; Bustamante, p. 179).

Por ello, de acuerdo con esta doctrina, en especial en el caso de responsabilidad médica, que es donde se viene presentando la mayor dificultad para la aplicación de la figura, no podría aceptarse la hipótesis de que el juez cuando acude a la pérdida de una oportunidad, lo hace por cuanto no está seguro del vínculo causal entre la muerte o las lesiones y la culpa del médico o cuando se duda de la adecuación del nexo causal entre esos extremos (lbíd., p.80,), habida cuenta que existe certidumbre sobre las causas de la privación de la oportunidad de vivir o de curarse, cual es la intervención del médico que priva al paciente de esa probabilidad "prevaleciente" (Parra, p.224). 
En suma, lo que se repara no es el haber truncado la obtención de la ganancia o el no evitar una pérdida, sino la posibilidad que se frustra, ese es el verdadero daño; pues como lo señala el profesor Chabas, lo que le da especificidad a esta figura, es el hecho de no identificar el perjuicio con la pérdida de una ventaja esperada sino con la privación de la oportunidad de obtenerla. (p. 69).

Comentando una sentencia de la primera Sala Civil de la Corte de Casación francesa del año de 1973, el profesor Chabas, nos ejemplifica lo que no debe entenderse como una pérdida de oportunidad:

Un cirujano no verifica que esté vacío el estómago del paciente cuya vida no está en peligro a corto plazo, pero que va a ser operado. El enfermo muere tal vez a causa de esa omisión, tal vez por causas naturales. El médico es condenado por haberle hecho perder las posibilidades (oportunidades) de sobrevivir.

Que hay de erróneo en esta sentencia? Primero que el paciente tenía todas sus posibilidades de sobrevivir al momento de la culpa médica. No puede decirse entonces que él sólo ha perdido oportunidades de sobrevivir. El ha perdido la vida. Es un punto esencial: para que pueda hablarse de pérdida de una oportunidad, se requiere que el enfermo no tuviera más que eso al momento de ocurrir la culpa médica. Pero se requiere además para que la 
condena sea concebible, que el vínculo de causalidad entre ese daño y culpa sea seguro, y es este es el segundo error de la sentencia: no se sabía si era por la culpa del médico que el paciente había perdido esas pseudo-oportunidades (p.82).

Señala el ilustre profesor, que afortunadamente semejante desviación de la pérdida de una chance, fue corregido posteriormente en una sentencia del año 1982, considerándose en dicha providencia que el administrador de justicia no puede conformarse con acudir a una condena por perdida de la oportunidad de sobrevivir cuando no alcanza a establecer un nexo de causalidad entre la culpa y la muerte, pues aquel es un perjuicio evaluable en función de las oportunidades de sobrevivir que tenía el paciente; por tanto, para condenar por esta clase de daños debe existir prueba de que la culpa médica ha sido la causante de la pérdida de esas oportunidades (p. 83).

\subsubsection{Clasificación de la teoría autonomista u ontológica}

Dentro de esta categoría existen dos tendencias: una, que considera el menoscabo producto de la pérdida de una chance, como una violación al patrimonio económico (teoría ontológica del perjuicio patrimonial); y otra, como una vulneración al patrimonio personal (teoría ontológica del perjuicio personal). 


\subsection{Teoría ontológica del perjuicio patrimonial.}

De acuerdo con esta teoría, la oportunidad perdida es un bien sui generis (no perceptible físicamente) de características económicas, preexistente al hecho dañoso e integrado al patrimonio de la víctima, su naturaleza es dinámica, destinado a desaparecer ya sea porque no logre actualizarse o porque se convierta en un beneficio real; por ello, el estudio de la causalidad no puede realizarse entre el hecho dañoso y la ventaja perdida- perjuicio final-, sino entre dicho hecho y la posibilidad de conseguir la ventaja esperada ( Medina, ps. 351 y $352 \mathrm{~A})$.

La teoría ontológica patrimonial, se divide a su vez en teoría ontológica moderada y teoría ontológica radical.

La teoría ontológica moderada, se aplica en eventos de incertidumbre causal donde no se tiene claridad si la víctima, de no haber ocurrido el hecho dañoso, habría obtenido el resultado benéfico esperado. En este evento, según sus partidarios, habría lugar a indemnizar parcialmente, toda vez que, como consecuencia del hecho perjudicial, de todas maneras, el afectado pierde definitivamente la oportunidad de lograr un beneficio, bien reparable, que antes de ser vulnerado hacia parte del patrimonio de aquel (Ibíd. p. 352).

Por esta vía, será reparable la oportunidad perdida que no sea aparente ni segura, es decir, las que tengan un grado de probabilidad de alcanzarse ubicadas entre un 
$15 \%$ y el umbral de certeza del $50 \%$ para el derecho anglosajón o el $80 \%$ para los países del continente europeo (Ibíd., p. 353). De tal suerte, que las oportunidades perdidas situadas en un rango inferior o en el umbral máximo de certeza, no se les aplica este sistema sino la figura del todo o nada.

La aproximación ontológica moderada, es posible identificarla dentro del contexto norteamericano bajo el nombre de "relaxed causation approach", el cual exige únicamente al demandante probar que perdió una oportunidad significativa de sobrevivir o recuperarse como consecuencia de la mala práctica médica; es decir, no requiere demostrar el porcentaje de probabilidad que supere el $50 \%$ exigido por la doctrina tradicional del todo o nada 6 .

Un ejemplo, de esta doctrina, es el caso Herskvtis v. Group Health Cooperative de la Suprema Corte de Washington. En este caso, un paciente fue equivocadamente diagnosticado y falleció como consecuencia de un cáncer de pulmón. A través de conceptos de expertos se demostró que la probabilidad de que el fallecido hubiera sobrevivido 5 años más era de un $39 \%$ al momento en que fue equivocadamente diagnosticado, pero solamente de un $25 \%$ al momento en que le fue diagnosticado correctamente el cáncer. Frente a esta situación, la

\footnotetext{
6 "the loss of chance theory is, essentially, one that allows an injured plaintiff to recover damages based upon a reduced standard of causation rather than the traditional one which requires the plaintiff to prove that it is more probable than not that the damage suffered was caused by the negligence of the defendant. . . [The relaxed causation approach] requires plaintiff to present evidence that a substantial or significant chance of survival or better recovery was lost. If plaintiff meets this initial threshold, the causation issue is submitted to the jury, using the traditional proximate cause standard to ascertain whether, in fact, the alleged malpractice resulted in the loss of a substantial or significant chance. Thus, the jury must find by a preponderance of the evidence that the alleged negligence was the proximate cause of the lost chance, but the lost chance itself need only be a substantial or significant chance, for a better result, absent any malpractice, rather than a greater than 50 percent chance of a better result." Marcella D. Borkowski, Administratrix (Estate Of Anthony Borkowski), Et Al. v. Chandra R. Sacheti, Appellate Court of Connecticut, 43 Conn. App. 294.
} 
Corte Suprema de Washington consideró que la conducta del demandado había disminuido las posibilidades de sobrevivir del paciente, lo cual era suficiente para considerar dicha situación como la causa del daño ${ }^{7}$.

En cuanto a la teoría ontológica radical, surge, según sus partidarios, para tratar de corregir las contradicciones en las que cae la tesis anterior al supeditar la existencia del bien económico pasible de resarcir por este recurso, al juicio de establecer si la posibilidad de alcanzar el beneficio son altas o muy vagas, desvaneciendo de este modo la existencia de ese bien del patrimonio jurídico del sujeto, en caso de ocurrir una cualquiera de esas situaciones (Ibíd., ps. 367 y 368).

Por ello, consideran que cualquier pérdida de una chance es indemnizable; su máximo inspirador ha sido el profesor King, quien considera que el daño actual no es la ventaja final sino la pérdida de la oportunidad; por tanto, la destrucción de las expectativas de alcanzar una ventaja, serán siempre indemnizables, esto es, cualquiera sea el rango de probabilidades que tenga la víctima. Esta teoría ha tenido escasa acogida por parte de la jurisprudencia al punto que los jueces de los Estados Unidos, al igual que los europeos no acostumbran reconocer indemnizaciones proporcionales cuando superan el umbral de certeza, en atención a que en estos eventos suelen optar por dar por cierto el hecho dañoso para

\footnotetext{
${ }^{7}$ Herskovits v. Group Health Cooperative, Supreme Court of Washington, 664 P.2d 474.
} 
condenar en consecuencia al pago de la indemnización total del perjuicio (Ibíd. ps. 368 y 369$)$.

En el derecho norteamericano la teoría ontológica radical se conoce con el nombre de "proportional approach" o "pure approach", la cual permite la obtención de daños y perjuicios a favor del demandante aún si éste tenía menos del $50 \%$ de posibilidades de recuperarse o sobrevivir. El demandante debe solamente probar que existió alguna negligencia que privó al paciente de la oportunidad de obtener o alcanzar una mejor recuperación. Por lo tanto, por ejemplo, bajo la forma pura, un paciente que tenga un $95 \%$ de posibilidades de morir, aun con un cuidado apropiado, tendría una acción contra el médico que negligentemente lo privó del $5 \%$ de recuperación (Wallace, p. 229). En consecuencia, la aproximación proporcional compensa a la víctima en proporción a su pérdida, sin importar si fue del 1\% 0 $99 \%$ (Zilich, p. 684).

\subsection{Teoría ontológica del perjuicio personal}

Bajo este enfoque se prescinde de inventar un bien económico inexistente para lograr el resarcimiento, pues se considera que la oportunidad de la cual goza una persona para alcanzar una ventaja, material 0 inmaterial, se encuentra directamente relacionada con el desarrollo de la personalidad; por ello, la pérdida de una chance no constituye una entidad económica, sino una situación vinculada con un bien espiritual, de tal suerte que el daño es el menoscabo de ese bien (Medina, p. 380). Un ejemplo, de esta postura, la podemos observar, en la 
jurisprudencia del Consejo de Estado de nuestro país, cuando al reflexionar sobre el perjuicio moral sufrido por los parientes de una persona a quien se le redujeron las probabilidades de sanar por omisión de la administración, señala que "...la pérdida de la oportunidad de sobrevivir, genera para las personas cercanas a la víctima un sufrimiento intenso, en la medida en que pierden también ellas la posibilidad de gozar de su compañía"8.

\subsection{Teoría de la causalidad probabilística}

De acuerdo con la responsabilidad proporcional se busca superar la injusticia que se produce bajo la teoría general de causación (todo o nada relajada), pues según esta última corriente, puede llegar a suceder que en eventos donde la causa es incierta se entienda probada cuando realmente no existe o al contrario, no se considere probada cuando en verdad existe. Adicionalmente, es injusto reconocer una indemnización de perjuicios completa en casos donde existe un $51 \%$ de posibilidades para generar el resultado dañoso y no reconocerlo en otro, donde existe sólo un $49 \%$, pues ambas situaciones son muy similares ${ }^{9}$.

En consecuencia, de acuerdo con esta doctrina, la mejor manera de compensar el daño es tomar en consideración la probabilidad de que el acto haya causado el

\footnotetext{
${ }^{8}$ Consejo de Estado, Sección Tercera, sentencia del 10 de febrero de 2000, Referencia No 11.878.

9 "No doubt this is a radical proposal, but it seeks to alleviate a radical injustice. When causation is uncertain there is always a chance that but for causation may be "proved" when in fact it does exist. Because proof does not require certainty in many cases, it is unfair to provide remedial relief as if it did. Furthermore, when the evidence shows a $51 \%$ chance of causation in one case and a $49 \%$ chance of causation in another, it is unfair to give full relief to the plaintiff in the former case and no relief in the latter. The two cases are practically the same"(Makdisi, p. 1065).
} 
daño y que los perjuicios reconocidos sean directamente proporcionales a esa probabilidad de causación.

Esta hipótesis es adoptada por el profesor Medina Alcoz, para fundamentar su teoría de la causalidad probabilística, como puede observarse en un trabajo donde analiza varios dictámenes del Consejo Consultivo de Castilla-La Mancha relacionados con reclamaciones de responsabilidad patrimonial contra el Servicio de Salud de dicha comunidad autónoma.

El profesor mencionado, manifiesta que centrar el fundamento de la pérdida de una chance, otorgando indemnizaciones parciales en eventos donde existe falta de prueba patente del nexo causal, es esconder la quiebra del dogma causal tradicional que representa la teoría de la perdida de una oportunidad, pues vincular, por ejemplo, la culpa médica con la privación de la oportunidad y no con el daño final, es apoyarse en artificios para afirmar su compatibilidad con la concepción tradicional de la causa. (p. 118 B).

Según el profesor mencionado, cuando se plantea para efectos de indemnizar que la probabilidad causal no es alta, sino simplemente seria, ello tiene como objetivo facilitar la protección que se estima debida, en tanto la víctima se privaría de una propiedad anterior (teoría ontológica del perjuicio patrimonial, desarrollada en Italia y en los Estados Unidos) o soportaría un daño moral (teoría ontológica del perjuicio personal, aplicada en Francia y, sobre todo, en España). Desde esta 
perspectiva se aprecia el perjuicio, a partir de la posibilidad de evitarlo y no desde el daño final incierto, sin parar mientes sobre el concepto tradicional de causa (lbíd.).

Se trata de construcciones doctrinales que no resisten un análisis crítico, pues dejan en manos del administrador de justicia, el determinar la existencia del daño. Así, si las circunstancias fácticas arrojan una probabilidad causal importante, se pronostica que el afectado, ha perdido un bien de naturaleza patrimonial o moral; pero si nuevos datos redefinen dicha perspectiva, arrojando una probabilidad muy alta o muy baja, aquel bien desaparece inexplicablemente para validar realmente la existencia o negación del nexo causal y, de esta suerte, conceder la reparación total o su rechazo (lbíd. p, 119 B).

Los casos de pérdida de oportunidad son difíciles y por tanto generan, por la forma como se vienen abordando, mucha incertidumbre, puesto que "surge la discordancia entre la solución que aporta el modelo tradicional (todo o nada, según que se rebajen o mantengan las exigencias ordinarias de prueba) y la que, según el sentir general, debería proporcionarse (indemnización proporcional)" ( $p$. 119 y120 B).

Para el profesor Medina Alcoz, tratar de compatibilizar la teoría de la perdida de una oportunidad con la teoría tradicional de la causalidad, con lleva a situaciones problemáticas, pues desde el punto de vista de esta última, lo lógico sería el 
rechazo de la primera, al exigir aquella, para efectos de indemnizar o no, la afirmación o negación del nexo causal; pero tal solución lastima la comprensión general de lo que es justo, en los eventos de daños posiblemente causados (p.120 B).

Por ello, propone, después de analizar el caso español, una reforma legal que facilitaría la labor del intérprete, donde se determinara con carácter general los supuestos donde se podría otorgar indemnizaciones parciales frente a causalidades posibles (lbíd.).

Aspectos de sensibilidad justicial, fuerzan "a admitir que, de la misma manera que la causalidad perfectamente demostrada genera una responsabilidad "total" (total liability) por el daño causado, la simplemente posible puede (sólo) en algunos casos generar una responsabilidad "parcial" (partial liability) por la posibilidad del daño causado" (p. 121 B).

De esta suerte, se redefine el concepto clásico de causalidad, dando cabida a la teoría de la pérdida de oportunidad, sin necesidad de acudir a ficciones, al establecerse una pauta de carácter general de responsabilidad proporcional mediante la cual se repara, "no por el daño que ha causado, sino simplemente por el daño que posiblemente causó" (Ibíd. p, 121 B). 
En esa línea, nos encontraríamos ante una teoría "con la que se afirmaría la existencia de una relación de causalidad entre el perjuicio (final) sufrido y la conducta del agente dañoso, pese a que ésta solo constituya una causa probable - meramente probable - de dicho perjuicio" (Medina p. 417 A).

\subsection{Teoría de la pérdida de oportunidad que excluye los casos de responsabilidad médica}

Para los profesores Prévot y Chaia, analizando en su parte introductoria el concepto de la pérdida de oportunidad de manera general con el fin de abordar el tema central del estudio, relacionado con la pérdida de chance de curación, señalan que en realidad lo malogrado son las expectativas de competir, participar, siendo estas las consecuencias mediatas previsibles y no las meras posibilidades de ganar, triunfar o aprobar, las cuales, en principio, no resultan atribuibles al victimario. Con todo, como esa oportunidad truncada revisada en su justa comprensión bajo la óptica de la fórmula tradicional del resarcimiento (daño material e inmaterial), generaría una insignificante reparación, se acude a la noción de chance como una especie de perjuicio autónomo (tertium genus), regido por un mecanismo de cuantificación especial, sujeto a la dúctil discrecionalidad judicial (ps. 49, 50 y 89 ).

Para los autores mencionados esta forma de concebir la pérdida de oportunidad, desborda la estructura causal de imputación de consecuencias, donde lo 
achacable al victimario es el haber privado a la victima la posibilidad de participar o competir, más no la probabilidad de haber obtenido el gran premio o ganado el certamen; pues de ser así, se conculcaría el régimen diseñado por el codificador decimonónico, conforme al cual la simple expectativa no es susceptible de indemnización (ps. 89, 90 y 91).

De esta suerte, se ha abusado de dicha institución, por cuanto se está indemnizando, realmente, es el daño tout court y no las consecuencias del evento causalmente imputables al victimario, al no advertir que la oportunidad cierta, merecedora de reparación, es el hecho de no haber participado, competido o concursado. Para ellos, no es la noción de pérdida de oportunidad lo criticable, sino la forma como se aplica, generando muchas veces, soluciones arbitrarias (lbíd., ps. 55 y 83).

Prévot y Chaia, a su vez, distinguen el problema de la perdida de una chance médica de la chance tradicional; señalando que en la primera, existe es una dificultad con respecto al nexo causal, al paso que en la segunda, "atañe al perjuicio, o más precisamente a la causalidad jurídica", es decir, el aprieto en este último caso, consiste en establecer la certeza del daño con relación a las consecuencias o efectos del evento (ps. 27 y 79 ).

En cuestiones de responsabilidad médica se conocen el hecho causante y el daño, siendo el nexo causal el elemento más incierto por la dificultad que, en 
ocasiones, se presenta de establecer, por ejemplo, si la muerte surgió de las "complicaciones sobrevenidas de la evolución normal de la enfermedad" o "del acto terapéutico falible". Esta posición, la comparte, el destacado profesor Tamayo, para quien en los casos de responsabilidad médica no se está en presencia de la pérdida de una oportunidad sino de una incertidumbre causal ( $p$. 363 tomo II)

Ante estos eventos, señalan los profesores Prévot y Chaia, que la noción de pérdida de una chance, es un instrumento "ingenioso y seductor artificio" de fácil aplicación (Ibid, p. 266), utilizado para aliviar los problemas de conciencia del juez (Tamayo, Tomo II, p. 363); permitiéndole mudar su inseguridad "sobre la relación de causalidad" a "una presunción de causalidad con daño disminuido" (Prévot y Chaia, p. 266).

Como el asunto a resolver en casos de responsabilidad médica, es una cuestión de corte causal, los doctrinantes mencionados consideran lo mejor, acudir a la figura de las "concausas" por ser la que más se acomoda al régimen del Código Civil, evitando de paso los riesgos que esconde la aplicación desmedida de la pérdida de una chance (Ibid, p. 267 y 271).

El fenómeno denominado concausa, se presenta cuando varias condiciones asumen el carácter de causas (Compagnucci p. 56) del resultado dañoso. En la responsabilidad médica, cuando hay coexistencias de causas que concurren al 
daño, esto es, el hecho de la victima (condiciones preexistentes del paciente) y la conducta del victimario (mala praxis médica), se produce una verdadera concausa, "cuya medida indemnizatoria será directamente proporcional a la incidencia causal de cada una" (Prévot y Chaia, p. 72). De allí, que el afectado no pueda exigir la reparación total del daño, pues éste sólo conseguirá un resarcimiento menor, teniendo en cuenta la incidencia causal de su hecho (Compagnucci p. 60).

Para aplicar la figura de las causas concurrentes, utilizan un umbral para distinguir los casos donde se debe reparar totalmente el daño de los casos en que se indemnizan parcialmente, como consecuencia de la concurrencia de causas. Así, para la primera situación, consideran debe establecerse un umbral de certeza que oscile entre el $51 \%$ (sistema angloamericano) o el $75 \%$ (jurisprudencia suiza) y para la segunda, los que estén por debajo de dicho umbral, sin llegar a ser menor a un porcentaje del $10 \%$ (criterio de los autores) o del $5 \%$ (jurisprudencia estadounidense) (lbíd., p. 275).

\section{Conclusiones}

Existen tesis a favor y en contra de la pérdida de una oportunidad algunas oponiéndose a su instauración como fórmula para reparar el daño; y otras, por el contrario, partidarias de su institucionalización con diferentes matices y vertientes. 
Con todo, si bien es un tema polémico y resbaladizo dada su negativa para aceptarla por algunos y las diferentes posturas de sus partidarios, se observa, al menos, una coincidencia en sus seguidores, como es la de fundamentar la tesis de reparar el perjuicio por razones de justicia en los casos donde por un tercero se ha perdido una oportunidad de obtener una ventaja o evitar una pérdida.

Las diferencias entre sus seguidores se centran básicamente en la forma de abordar el problema, pues mientras algunos lo refieren a un problema de incerteza respecto al daño, o como lo define el profesor Chabas, de incertidumbre inherente al perjuicio (teoría ontológica) otros, lo identifican como un problema de nexo causal fáctico (Teoría de la causalidad probabilística).

Hay otra teoría, que podríamos llamar mixta, que acepta la primera postura para casos diferentes a la responsabilidad médica; y para ésta, la segunda, por ser una cuestión de corte causal (teoría de la concurrencia de causas).

De las posturas propuestas siendo todas tan racionales, consideramos, a pesar de tener una acogida minoritaria, la más equitativa frente a los intereses tanto de la victima como del victimario, a la teoría moderada de la causalidad probabilística, la cual al redefinir el concepto clásico de causalidad, sin utilizar ficciones, permitiría aplicar, sin excepción, a todos los casos, la teoría de la pérdida de una oportunidad imponiendo una responsabilidad al victimario acorde al verdadero daño causado. 
Si bien no se tiene la certidumbre de que la actuación u omisión del victimario haya dado lugar a que el afectado hubiese obtenido la ventaja o evitado la pérdida, si es claro que por su actividad se impide conocer de manera definitiva si la victima habría alcanzado el resultado anhelado; situación que en caso de generar algún perjuicio, este podría establecerse teniendo en cuenta el grado de probabilidad causal que haya alcanzado su actuación respecto al resultado final.

Así, desde este prisma, la pérdida de una oportunidad no tendría ninguna aplicación en los casos donde pueda emplearse la teoría del todo o nada sin ninguna hesitación, quedando para aquellos eventos de incertidumbre causal, es decir, para las situaciones no cobijadas por aquella teoría, en atención a que las probabilidades de causalidad, si bien son insuficientes para dar por cierto el daño causado, no pueden ser descartadas, por razones de justicia para responsabilizar y obligar, bajo la teoría de la perdida de oportunidad, al pago de una indemnización parcial dado el grado de probabilidad causal sustancial existente, el cual se puede establecer acudiendo a las reglas de la matemática, la estadística y la lógica según el evento o, a las reglas de clausura en caso de dudas resultantes de un cálculo de probabilidades confusas o poco concluyente ( principio pro damnato y la carga de la prueba). 


\section{Referencias}

Bonasi, Eduardo. (1958). La Responsabilidad Civil. Barcelona: José M. Bosch.

Bustamante, Jorge. (1997). Teoría General de la Responsabilidad Civil. Buenos Aires: Abelardo Perrot.

Compagnucci, Rubén. (1984). Responsabilidad Civil y relación de causalidad, en Seguros y Responsabilidad Civil No. 5. Buenos Aires: Editorial Astrea.

Chabas, Francois. (2000). La Pérdida de una Oportunidad (Chance) en el Derecho francés de la Responsabilidad Civil. Revista de Responsabilidad Civil y del Estado, Instituto Antioqueño de Responsabilidad Civil y del Estado.

De Cupis, Adriano. (1975). El Daño. Barcelona: Bosch.

Karcher, Richard T. (2010). Rethinking Damages for Lost Earning Capacity in a Professional Sports Career: How to Translate Today's Athletic Potential into Tomorrow's Dollars. Chapman Law Review. Volumen 14. p. 75.

Makdisi, John. (1988-1989). Proportional Liability: A Comprehensive Rule to Apportion Tort Damages Based on Probability. Nort Carolina Law Review. Volumen 67. p.1063. 
Mazeaud, Henri, Mazeaud León y Tunc, André. (1961). Tratado Teórico y Práctico de la Responsabilidad Civil delictual y contractual. Buenos Aires: Ediciones Jurídicas Europa América.

Medina, Luís. (2007a). La teoría de la Pérdida de Oportunidad. Thomson.

Medina, Luís. (Diciembre 2009b). La Doctrina de la Pérdida de oportunidad en los Dictámenes del Consejo Consultivo de Castilla- La Mancha. Reflexiones Críticas. Revista jurídica de Castilla - La Mancha, No. 47.

Merryman, Jhon. (2008). La tradición jurídica Romano- Canónica. Fondo de Cultura Económica. Undécima reimpresión.

Parra, Jairo. (2011). Dificultades de la Prueba del nexo Causal ante la pérdida de un Chance. En Responsabilidad Civil y Negocio Jurídico. Bogotá: Editorial Ibáñez y Universidad Santo Tomás.

Peirano, Jorge. (2004). Responsabilidad Extracontractual. Bogotá: Editorial Temis.

Prévot, Juan y Chaia, Rubén. (2007). Pérdida de Chance de Curación. Buenos Aires: Editorial Astrea.

Tamayo, Javier. (2007). Tratado de Responsabilidad Civil. Tomo 1. Bogotá: Editorial Legis. 
Tamayo, Javier. (2007). Tratado de Responsabilidad Civil. Tomo 2. Bogotá: Editorial Legis.

Trigo, Félix. (2008). Pérdida de Chance. Buenos Aires: Editorial Astrea.

Villey, Michel. (1979). Compendio de Filosofía del Derecho. Pamplona: Universidad de Navarra.

Wallace, Brie D. (2009). Poor Policy Stunts Tennessee Tort Law Again: The Need for Tennessee's Adoption of the Loss of Chance Doctrine in Medical Malpractice Litigation. University Of Memphis Law Reviw. Volumen 40. p. 215.

Yzquierdo, Mariano. (2001). Sistema de Responsabilidad Civil Contractual y Extracontractual. Madrid: Editorial Dykinson.

Zannoni, Eduardo. (1982). El Daño en las Responsabilidad Civil. Buenos Aires: Editorial Astrea.

Zavala de González, Matilde. (2009). Tratado de Daños a las Personas Disminuciones Psicofísicas-. Editorial Astrea. 
Zilich, George J. (2002-2003). Cutting through the Confusion of the Loss-OfChance Doctrine under Ohio Law: A New Cause of Action or a New Standard of Causation?. Cleveland State Law Reviw. Volumen 50. p. 673. 